\title{
Clinical Application of Resorbable Polymers in Guided Bone Regeneration
}

\author{
Wing Yuk Ip and S. Gogolewski \\ Department of Orthopaedic Surgery, The University of Hong Kong, Hong Kong SAR, \\ Polymer Research, AO Research Institute, Davos, Switzerland
}

INTRODUCTION: Long segmental diaphyseal bone loss often results from high energy trauma like blast injury, osteomyelitis or wide excision of malignant conditions. Treatment of these long segmental diaphyseal defects remains a difficult clinical problem. In the literature, many authors have reported that segmental bone defects longer than $2.5 \mathrm{~cm}$ always require bone grafting. Nonvascularized bone graft frequently fails if the defect is longer than $6-7 \mathrm{~cm}$. Thus, a $2.5 \mathrm{~cm}$ long bone defects is probably the critical-size defect in human and a $7 \mathrm{~cm}$ long defect is likely the criticalsize for non-vascularized bone graft.

Various treatment methods are adopted currently to address this problem, including vascularized bone graft, distraction osteogenesis and massive allograft. However, all these methods are associated with a lot of problems.

Successful guided bone regeneration has been achieved in skull bone and jaw bone using resorbable allograft. Bone regeneration in long segmental defect and relatively small defect in tumour excision has been achieved using resorbable polylactide scaffolds.

METHODS \& MATERIALS: Bioresorbable scaffolds (microporous membranes and 3-D sponges) were produced from poly(L/DL-lactide) $80 / 20 \%$ with a molecular weight of $200 \mathrm{kD}$ (PURAC Biochem; Gorinchem, The Netherlands). The membranes had interconnected open pores with an average size of 50 to 70 $\mu \mathrm{m}$. The 3-D sponges had the average pore size in the range of $450-700 \mu \mathrm{m}$, and the pore-to-volume ratio in the scaffold $94 \%$.

Ten patients with bone defect of sizes up to 6 $\mathrm{cm}$ due to various causes including benign tumour, osteomyelitis \& fractures were treated with resorbable polylactide scaffold impregnated with marrow blood which contains stromal cells. In cases with infection, antibiotics was also loaded into the scaffold and in this situation, the scaffold also served as a drug delivery device. The patients have been assessed regularly with X-rays and clinical symptoms.
RESULTS: Serial X-ray evaluation and clinical evaluation revealed presence of bone regeneration. The limbs enjoyed satisfactory function and there was minimal donor site morbidity and major surgery can be avoided.

DISCUSSION AND CONCLUSIONS: Segmental bone defects in selected clinical cases were treated using bioresorbable porous polymeric scaffolds impregnated with autogenous marrow blood. Such cases would be treated otherwise by conventional techniques.

Vascularized bone transfer has limited supply and involves a major operation. There is always a chance of vascular complication and there is donor site morbidity. There is a limitation in bone defect size that can be lengthened using distraction osteogenesis, and in addition, this procedure requires a prolonged placement of an external fixation. There is a high chance of traction injury to nerve and other soft tissues.

Massive allograft requires a prolonged period, in terms of decades, for complete creep substitution. There is also a high incidence of disease transmission and infection.

Therefore there is a constant demand for bone substitute which can bridge long segmental defect effectively with minimal morbidity and can heal in reasonable time frame. The affected limb can be rehabilitated and bear weight for functional restoration as early as possible. If such a substitute is radiolucent this would be an additional advantage for a radiographic assessment of bone healing.

Such a new bone substitute can be for example produced from bioresorbable polymers and/or composites consisting of bioresorbale polymers and calcium phosphate ceramics which would add and osteoconductive component to the implant. Impregnation of such bone substitutes with autogenous bone marrow or marrow blood would also make osteoinductive. The early promising results of this study show that such an approach to new bone substitutes is feasible. 